\title{
Flexible Architecture Software Radio OFDM Transceiver System and Frame Synchronization Analysis
}

\author{
Keith Nolan, Philip Mackenzie, Linda Doyle, Declan Flood \\ Networks and Telecommunications Research Group \\ Electronic and Electrical Engineering Department \\ Trinity College Dublin \\ Rep. of Ireland \\ \{nolanke, mackenp, ledoyle,floodk\}@ @cd.ie
}

\begin{abstract}
In this paper, we present findings relating to frame synchronization algorithms for software radio based OFDM systems in addition to an overview of the design and implementation of an (OFDM) Software Radio based on a general-purpose processor (GPP) platform. The objectives of this work are to advance on existing research carried out in the area of reconfigurable wireless flexible architecture nodes, which are subject to multi-path fading, Doppler effects, and adjacent channel interference and have low cost yet robust and reliable operation requirements. We also include findings dealing with Bit Error Rate (BER) versus channel Signal to Noise Ratio (SNR) for BPSK and QPSK modulation schemes, and also the effect of non real-time Operating Systems (OS) running on GPPs on the transmitter processing time.
\end{abstract}

\section{INTRODUCTION}

There has been a dramatic move towards replacing analogue radio components with Digital Signal Processors and digitizing $\mathrm{RF}$ signals as close as possible to the antenna. Radio system design is greatly simplified using this approach, since typical hardware radio components are replaced by software implementations. Future generations of wireless networks will take advantage of software realizations of hardware devices because of the highly re-configurable nature of such systems allowing the implementation of many different modulation schemes on a single hardware system [1][2][3]. This paper presents a novel approach for a multi-carrier software radio implementation of an OFDM receiver. This will facilitate the creation of an adaptive OFDM transceiver system capable of dynamically changing system parameters in order to maximize channel capacity usage and minimize power consumption based on the user requirements and channel capacity.

OFDM (Orthogonal Frequency Division Multiplexing), used for digital audio and video broadcasting (DAB, DVB) is now accepted as the standard for next generation wireless LAN systems. The rapid increase in popularity for this scheme is due to the high spectral efficiency and high data rate capability of OFDM transceiver systems over broadband radio channels. Multi-carrier OFDM systems have the ability to combat frequency-selective fading, multi-path, and jamming effects for both fixed and mobile wireless nodes. In mobile node circumstances, fast fading, shadowing and path loss causes frequency and time-dispersion but OFDM offers a solution for these problems using sub-carrier information bits and channel coding.

Three major problems present themselves when dealing with OFDM transceiver systems; one is that the OFDM symbol arrival time is unknown and must be determined. Multi-carrier systems are much more sensitive to time offsets than single carrier systems due to the necessity for multi-carrier signal orthogonality. The second major problem is that there may be a mismatch between the transmitter and receiver oscillators. Demodulation of a signal with a carrier frequency offset results may result in a loss of signal orthogonality and thus lead to data errors as well as degrade the performance of the symbol synchronization algorithm. Thirdly, the peak-to-average power (PAPR) of an OFDM signal is quite high and can lead to nonlinear transmitter amplifier operation. The flexible-architecture OFDM system outlined in this paper facilitates automatic PAPR reduction algorithm selection in addition to hardware-free symbol and frequency synchronization techniques.

Several papers in the last few years have focused on hardware implementations of OFDM systems and synchronization issues. This paper deals specifically with a flexible architecture OFDM system using optimum synchronization techniques for adaptable transceiver design and rapid prototyping Coulson [4][5] presents an analysis of OFDM maximum likelihood synchronization algorithms using a pilot symbol and provides analyses of method used to determine suitable detection threshold values. Seo, Kim, Park, and Ihm [6] approach optimum synchronization techniques using two OFDM training symbols. One training symbol is used for symbol timing recovery and the second symbol is used to estimate the carrier frequency offset. A pseudo-noise (PN) sequence provides the data source for the even sub-carriers and the odd sub-carrier values are zero.

Schmidl and Cox [7][8] also examine the use of a training sequence of two OFDM symbols to correct the carrier frequency offset and estimate the start of frame position. Each training symbol has two identical halves. After passing through the 

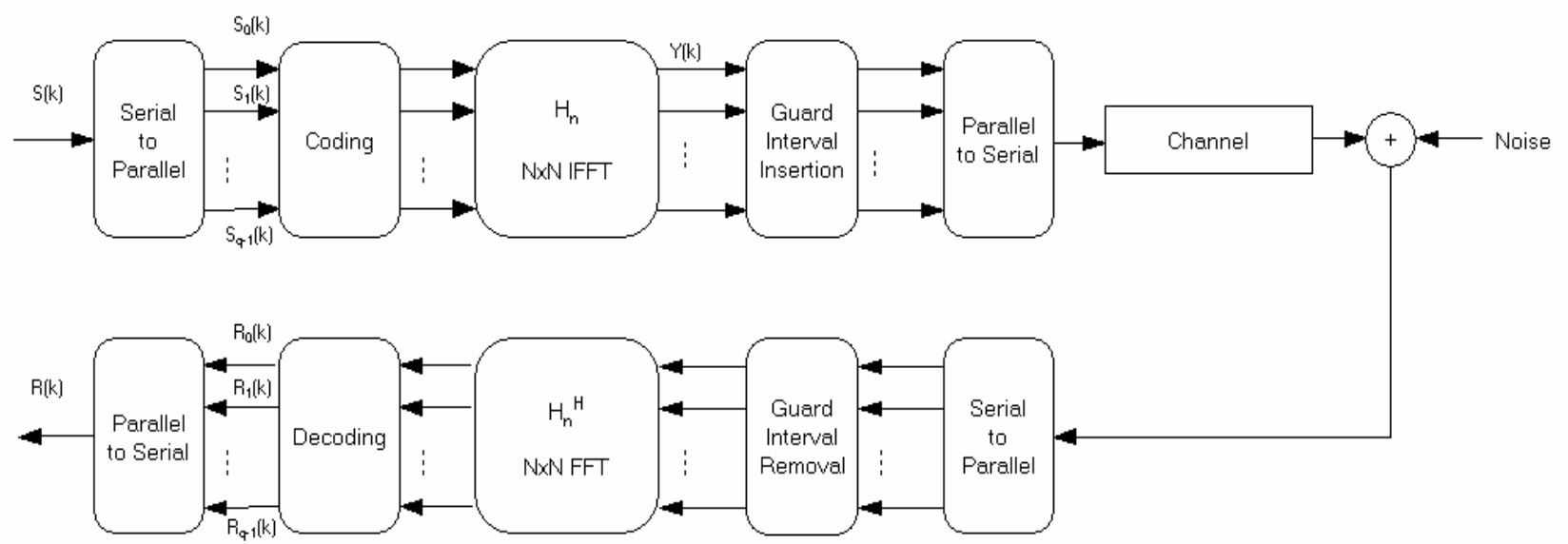

Figure 1: Block diagram of an OFDM transceiver system

channel, the carrier frequency offset may be calculated from the phase difference between the two symbol halves.

\section{SOFTWARE RADIO SYSTEM}

Fig.2 outlines a typical software radio system, which is a transceiver that has a hardware RF front-end but the modulation/demodulation functions are carried out in software. The RF front-end amplifies the signal from the antenna, converts the intercepted pass-band signal to an intermediate frequency (IF), and performs an initial stage of signal filtering if required. The analogue-to-digital converter (ADC) digitizes the filtered received signal and the software-processing core performs passband to base-band conversion and demodulation [9]. The IFFT block size, sub-carrier modulation scheme, source (and data sink) files, carrier power levels and other internal functions such as signal windowing and header power may be configured using a radio XML based configuration tool called IRIS (Implementing Radio in Software), which has been developed by this research group over the past three years. [10][11][12] The IRIS system configures the software radio at run-time allowing the rapid deployment of many different transceiver styles for testing and remote radio configuration. For the transmitter; the air-interface is the digital-to-analogue converter (DAC), the base-band OFDM signal is converted to pass-band using an up-converter to the desired working frequency range.

The OFDM transceiver system presented in this paper is a flexible architecture radio system based on the IRIS software radio platform. This combines both software tools and a development environment that can be used to develop adaptable wireless implementations. In particular, IRIS was created to provide an environment that allows for the development and experimentation of new and exciting wireless communication techniques. We begin by outlining the OFDM signal generation process, demodulation, and how this system is implemented as a software radio.

\section{OFDM GENERATION \& IFFT}

Consider an OFDM transceiver system with $N_{S C}$

Sub-carriers used for transmission, using a FFT of length $N_{F F T}$. The OFDM signal is generated at base-band by performing an Inverse Fast Fourier Transform (IFFT) on the complex-valued sub-symbols as follows:

$x(n)=\frac{1}{N_{F F T}} \sum_{k=0}^{N_{F F T} 1} x(k) \exp \left\{j 2 \pi \frac{n k}{N}\right\} ; n=0,1, \cdots, N_{F F T}-1$

An OFDM signal is composed of a training sequence followed by the payload data. The purpose of the training sequence is to facilitate symbol-timing recovery, carrier frequency

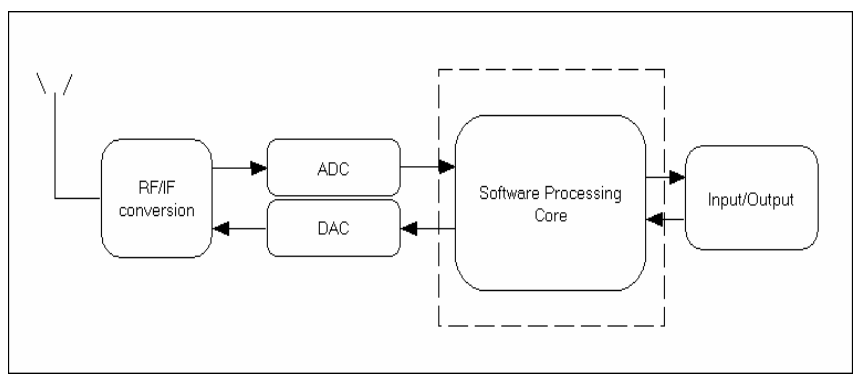

Figure 2: Block diagram of a Software Radio System 
synchronization, and channel response estimation. An OFDM symbol has a guard interval and a data period. The actual transmitted signal sequence is $\left\{\begin{array}{l}x\left(N_{F F T}-N_{G I}\right), x\left(N_{F F T}-N_{G I}-1\right), \ldots, \\ x\left(N_{F F T}-1\right), x(0), X(1), \ldots x\left(N_{F F T}-1\right)\end{array}\right\}$,

This sequence is prefaced with the guard interval $N_{G I}$, which is longer than the channel impulse response to ensure that intersymbol interference (ISI) is minimized. After transmission over a multi-path channel, the receiver samples are

$r(n)=\sum_{k=0}^{k=N_{s}-1} x(n-k) \cdot h_{m}(k)+w(n)$

where $h_{m}(k)$ is the complex channel impulse response sampled at the receiver and $w(n)$ is complex-valued zero-mean Additive White Gaussian Noise (AWGN) with a variance of $2 \sigma_{w}^{2}$. We assume that the channel is slowly time varying and the channel impulse response remains constant during the symbol period.

The guard interval must be removed prior to demodulation. The demodulation process consists of performing a FFT on the received signal (with guard interval removed correctly) resulting in the received sub-carrier symbols. The $k$-th element of the resultant FFT sequence may be expressed as

$$
Y(k)=\sum_{n=0}^{N_{F F T}-1} r(n) \exp \left\{-j 2 \pi \frac{n k}{N}\right\}
$$

\section{FRAME SYNCRONIZATION}

Symbol timing recovery involves searching the time domain signal for a training symbol with two identical halves. After passing through the transmission channel, the symbol will remain identical except that a phase difference between the two halves will be present due to the carrier frequency offset. A pseudo-noise (PN) sequence is transmitted on the even frequencies and zeros are transmitted on the odd frequencies. This method is used to make the two symbol halves identical in time. Using a PN sequence, one of the points of a M-aryPSK constellation is transmitted and to ensure that approximately constant high signal energy is maintained for each training symbol, the symbol frequency components are multiplied by $\sqrt{2}$ prior to transmission.

In practical terms, a set of constellation points for one half of the training symbol are determined using the PN sequence and then repeated for the second half of the symbol.

Training symbols are distinguished from actual data symbols in the received signal sequence from the fact that an actual data symbol does not contain null-valued odd frequency sub-carriers. A second training symbol containing a PN sequence on the odd frequencies to determine the sub-channel delays, and another PN sequence is used on the even frequencies in order to estimate the carrier frequency offset. An important issue when dealing with OFDM systems is that the peak-to-average power ratio (PAPR) is high. This can result in non-linear amplification effects leading to distortion at the transmitter amplifier. Two criterion therefore for using PN sequences are that the sequence is easy to implement and that they have a low PAPR in order to minimize distortion. The received signal is split in two by multiplying it by values from a $\sin / \cos$ lookup table resulting in both in-phase and quadrature base-band components. The use of a GPP eliminates the need for an analog local oscillator (LO) or numerically controlled oscillator (NCO), thus, a further reduction of hardware requirements.

With regards to time ordering, the first half of the first training symbol is identical to the second half, except for a phase difference caused by the carrier frequency offset. The training symbol detection algorithm operates by multiplying a conjugate value of a sample from the first training symbol half by the corresponding sample from the second half. This latter sample is received $T / 2$ seconds later. The effect of the channel on the received symbol should cancel, and the result will have a phase difference of approximately $\varphi=\pi T \Delta f$. When the start of the frame is detected i.e. the training symbol is received, the products of each of these sample pairs will have approximately the same phase because we assume that the channel impulse response remains constant during a symbol period. This means that the magnitude of the sum will be significantly larger on detected of a training symbol. Schmidl and Cox present in [7][8], an algorithm called Maximum-Normalized Correlation timing and Carrier Frequency Offset (CFO) estimation. The timing offset is found by maximizing

$\hat{m}_{\max }=\arg \max _{k}\left(\frac{|\psi(k)|^{2}}{P(k+L)^{2}}\right)$

where $\psi(k)=\sum_{j=k}^{k+N_{G I}} r(j) r^{*}(j+N)$

is the complex correlation between $N_{G I}$ samples $N$ samples apart and

$P(j)=\sum_{j=k}^{k+L-1}|r(j)|^{2}$ 
TABLE 1

EXAMPLE PN SEQUENCE FOR A TRAINING SYMBOL USING 11 SUB-CARRIERS

\begin{tabular}{|r|r|r|}
\hline Freq. No. $\mathrm{k}$ & $\mathrm{S}_{1}, \mathrm{k}$ (sym half 1$)$ & $\mathrm{S}_{2}, \mathrm{k}$ (sym half 2$)$ \\
\hline-5 & $7-7 \mathrm{j}$ & $4+4 \mathrm{j}$ \\
-4 & 0 & $-4-4 \mathrm{j}$ \\
-3 & $7+7 \mathrm{j}$ & $4-4 \mathrm{j}$ \\
-2 & 0 & $-4+4 \mathrm{j}$ \\
-1 & $-7-7 \mathrm{j}$ & $4-4 \mathrm{j}$ \\
0 & 0 & $-4-4 \mathrm{j}$ \\
1 & $-7+7 \mathrm{j}$ & $4+4 \mathrm{j}$ \\
2 & 0 & $-4+4 \mathrm{j}$ \\
3 & $7+7 \mathrm{j}$ & $4-4 \mathrm{j}$ \\
4 & 0 & $-4+4 \mathrm{k}$ \\
5 & $7+7 \mathrm{j}$ & $4+4 \mathrm{j}$ \\
\hline
\end{tabular}

\section{IMPLEMENTATION AND GPP ISSUES}

Fig.1 shows the block diagram of a GPP based transceiver. Considering the transmitter firstly, the serial input stream is converted to a stream of symbols, and then the serial-to-parallel converter results in $\mathrm{N}$ parallel streams of length $\mathrm{M}$, where $\mathrm{N}$ is the number of sub-carriers used and $\mathrm{M}$ is the total number of input symbols divided by the number of carriers. Each parallel stream is modulated using the desired modulation technique e.g. DQPSK for DAB, and then each sub-carrier is inserted into the overall spectrum. The normalized OFDM signal is converted to an analogue signal and passed to the up-conversion stage [13]. Table 1 shows an example of a PN sequence used for one 11 sub-carrier training symbol.

The signal of interest is sourced from the IF output of an AOR 8600 receiver. After band-pass filtering and amplification, the IF signal is under-sampled using a 12-bit 20Msamples/sec PCI card ADC operating at $4 \mathrm{MS} / \mathrm{sec}$. A $2 \mathrm{GHz}$ Pentium III forms the software-processing core and the processing software is created using a high-level language development environment $(\mathrm{C}++)$.

\section{RESULTS}

The OFDM system outlined in this paper using IRIS permits modification of IFFT block size, number of symbols per frame and sub-carrier modulation scheme selection in order to maximize the ratio of transceiver performance to power consumption. In this section, we firstly examine the frame synchronization metric values for symbol sample offsets ranging from zero (perfect synchronization) to FFT size (zero synchronization). Secondly, we examine the Bit Error Rate (BER) versus the channel Signal to Noise Ratio (SNR) where the sub-carrier modulation schemes are BPSK (Binary Phase Shift Keying) and (QPSK) Quadrature Phase Shift Keying respectively. The channel SNR is varied from $100 \mathrm{~dB}$ to $0 \mathrm{~dB}$,

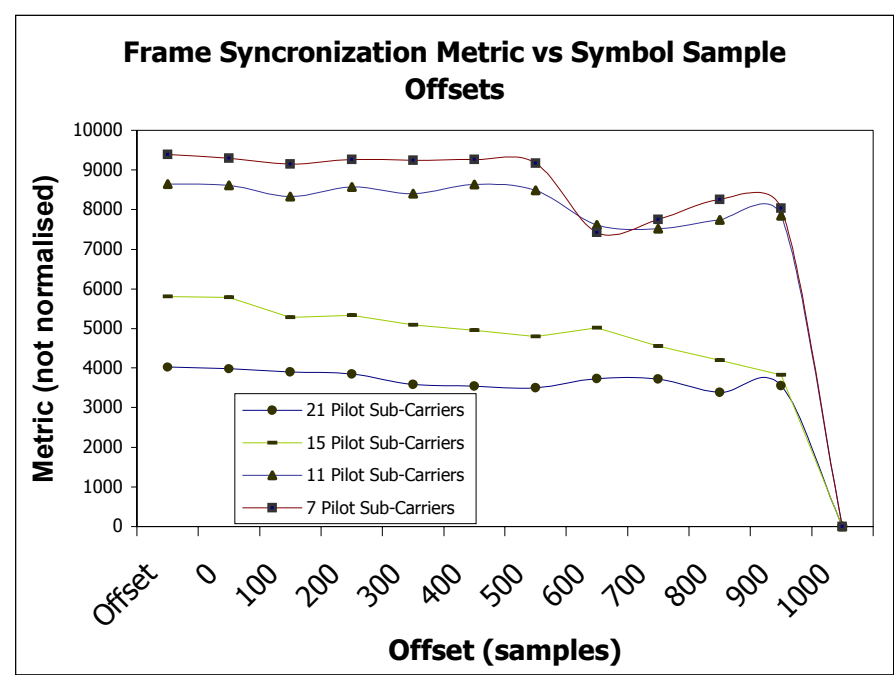

Figure 3: Graph of symbol sample offsets versus the frame synchronization metric

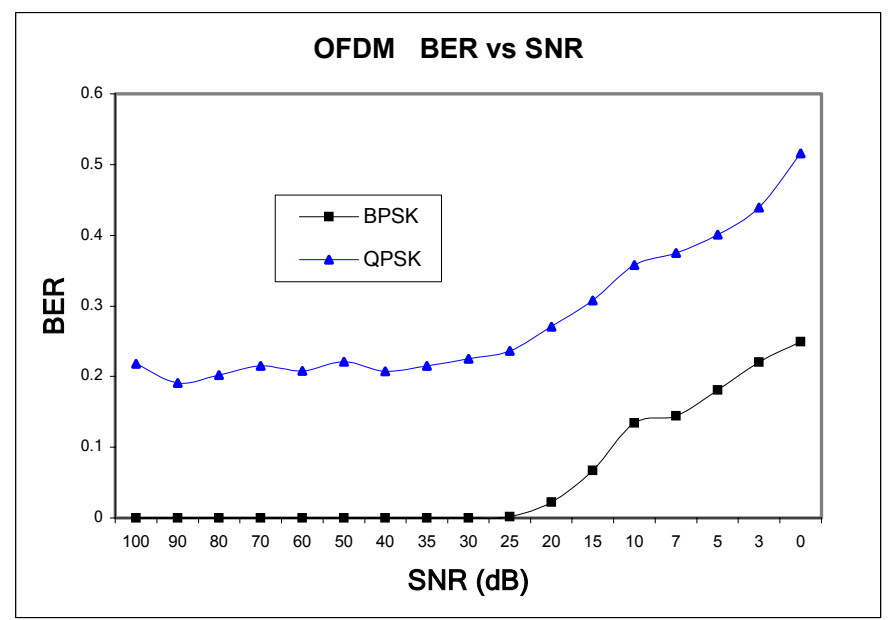

Figure 4: Graph of Bit Error Rate (BER) Vs. Channel SNR

100 OFDM Frame Generation Processing Time (ms)

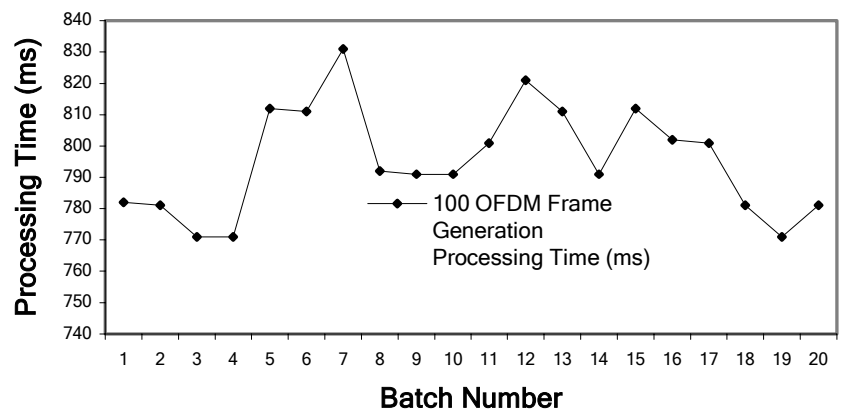

Figure 5: Graph showing the variation of OFDM frame generation times for 20 batches 
the channel is corrupted by AWGN, and the duration of each symbol is one sample/symbol. Fig. 3 shows that the synchronization metric peaks for a zero timing offset situation and tends towards zero as the offset approaches the FFT length.

This test series is for a pilot training sequence of five, ten, fifteen and twenty sub-carriers.

The highest metric values occur for the twenty pilot sub-carrier case due to the expected high sum of products value. A dramatic fall off occurs as the offset approaches the FFT length. Fig. 4 shows that for the QPSK case, the constraint of one sample per symbol results in a high BER at higher SNR values while for the BPSK case, the BER is zero until the channel SNR is reduced to approximately $30 \mathrm{~dB}$. For a low power mobile node with realtime operation objectives, the processing time overhead needs to be minimized

Unlike dedicated DSPs, general-purpose processors, using nonreal-time operating systems do not offer fully deterministic processing times due to the CPU thread scheduling for the different services that are running on the PC. Fig. 5 shows how the processing time varies for several 100 OFDM frame generation runs. For this twenty runs example, the processing time varies within the range $[771 \mathrm{~ms}, 831 \mathrm{~ms}]$. In order to meet the real-time operation objectives, a sufficient processing time overhead must be allowed for the maximum processing time measured.

\section{CONCLUSION}

This paper has demonstrated that a re-configurable low-cost and low-power consumption OFDM wireless node is possible using general-purpose processors. Our findings indicate that pilot symbols using relatively few sub-carriers is suitable for start of frame detection thus greatly reducing the processing time overhead. The experimental results from an actual implementation also show that a highly optimized software realization of an OFDM transceiver is capable of meeting realtime operation objectives. The $\mathrm{ADC} / \mathrm{DAC}$ rates effectively set the possible channel bandwidth so for reliable wideband OFDM signal reception and generation, it is vital to attain very high rate devices. Pass-band sampling demands extremely high ADC rates hence the need to convert the intercepted signal to a lower intermediate frequency but the ADC/DAC rates must have a sufficient rate to reliably sample/output the entire OFDM channel bandwidth. The next stage of this work is to combine a flexible architecture OFDM transmitter capable of being dynamically modified based on channel information from the receiver to maximize channel capacity usage and minimize power consumption. Further work is also being carried out on optimized waveform generation techniques and the incorporation of modulation scheme recognition techniques into the OFDM system with the aim of developing an adaptive modulation system.

\section{ACKNOWLEDGMENT}

This work was carried out with the aid of an IRCSET Government of Ireland grant.

\section{REFERENCES}

[1] Michael Ismert. Making Commodity PCs Fit for Signal Processing. In USENIX. USENIX, June1998.

[2] Vanu G. Bose, Michael Ismert, Matthew Welborn, and John Guttag. Virtual Radios. JSAC issue on Software Radios, February 1999.

[3] PC-based receiver for Eureka-147 digital audio broadcasting, Cho, J; Cho, N; Bang, K; Park, M; Jun, H; Park, H; Hong, $D$., Broadcasting, IEEE Transactions on, vol. 47 Issue: two, June 2001, pp. 95-102

[4] Maximum Likelihood Synchronization for OFDM Using a Pilot Symbol: Analysis, Coulson, A.J.; IEEE Journal on Selected Areas in Communications, Vol. 19, No. 12, December 2001, pp2495-2503

[5] Maximum Likelihood Synchronization for OFDM Using a Pilot Symbol: Algorithms, Coulson, A, J.; IEEE Journal on Selected Areas in Communications, vol. 19, No 12,December 2001,pp2486-2494

[6] Fast and low-complex frequency synchronization for OFDM Bo-Seok Seo; Su-Chang Kim; Jinwoo Park; Jong-Tae Ihm; Vehicular Technology Conference, 2001. VTC 2001 Fall. IEEE VTS 54th, Volume: 2, 2001 pp: 1120 -1124 vol.2

Low-overhead, low-complexity [burst] synchronization for OFDM Schmidl, T.M.; Cox, D.C.; Communications, 1996. ICC 96, Conference Record, Converging Technologies for Tomorrow's Applications. 1996 IEEE International Conference on, Volume: 3, 23-27Jun 1996 pp: $1301-1306$ vol.3

[8] Robust frequency and timing synchronization for OFDM Schmidl, T.M.; Cox, D.C.; Communications, IEEE Transactions on, Volume: 45 Issue: 12, Dec 1997 pp: $1613-1621$

[9] Eyre, J.: "The Digital Signal Processing Derby", IEEE Spectrum, vol. 38 , no. 6, pp. 62-68, Jun 2001

[10] IRIS: A test-bed for developing re-configurable software radio, Mackenzie, P; Doyle, L; Nolan, K; Flood D.; unpublished

[11] Selecting Appropriate Hardware for Software Radio Systems, Mackenzie, P., Doyle, L., Nolan, K., O'Mahony, D. to appear in the proceedings of 2002 Software Defined Radio Technical Conference

[12] A Component Based Software Radio Engine Using XML, Mackenzie, P., Doyle, L., Nolan, K., O'Mahony, D. to appear in the proceedings of the 5th International Symposium on Wireless Personal Multimedia Communications

[13] Performance Of FFT Processor in Future OFDM System, Chai, K.K., Loo, K.K., Alukaidey, S., Jimaa. A., Salman, K., Proceeedings of the IASTED International Conference on Signal Processing, Pattern Recognition \& Applications, June 25-28, 2002, pp 7-12 